\title{
(Zeit)Befindlichkeit und partieller Gleichlauf in der Sprache. Eine Untersuchung zum Deutschen
}

\author{
Temporal Relations of partial simultaneity \\ in adverbial clauses in German \\ Mariola WIERZBICKA ${ }^{1}$ \\ Uniwersytet Rzeszowski
}

\begin{abstract}
The paper discusses ways of expressing the temporal relations of partial simultaneity in adverbial clauses in the German language. Although the relations can be expressed by participle phrases, noun phrases, prepositional phrases and infinitive phrases, the adverbial clause is the most frequent means of expressing the relations. The temporal adverbial clause has an almost unlimited range of applications, which stretches from vaguely hinted relation to absolute necessity, and from general statements and clarifications to definite emotionally motivated utterances. Wherever there is an obvious connection between facts, events, actions, relations as well as personal will and feeling, it can be expressed by means of a temporal structure. The subject of the paper is the influence of conjunctions während, als, wenn, seit(dem) and solange on the time arrangement of situations introduced into the time clause and the main clause with regard to morphological, syntactic and semantic elements and dependence on the relation of the correspondence between events in the time clause and events in the main clause in German.
\end{abstract}

Keywords: temporal relation, temporal clauses, partial simultaneity

1 (D) https://orcid.org/0000-0003-2125-0204. 


\section{Zusammenfassung}

Der vorliegende Beitrag diskutiert den sprachlichen Ausdruck von Relationen der partiellen Gleichzeitigkeit im Deutschen. Obwohl es zur Bezeichnung temporaler Beziehungen eine Liste von Möglichkeiten gibt, wie Partizipial-, Nominal-, Präpositional- oder Infinitivphrasen, ist das eigentliche Temporalsatzgefüge das bei weitem häufigste temporale Ausdrucksmittel. Das Temporalsatzgefüge hat nahezu unbeschränkten Anwendungsbereich, der von dem fast nur angedeuteten Zusammenhang bis zur zwingenden Notwendigkeit, von Allgemeinaussagen und didaktischen Erläuterungen bis zu emotional bestimmten Motivationen reicht. Wo immer ein temporaler Zusammenhang zwischen Tatsachen, Ereignissen, Handlungen und Verhältnissen oder auch zwischen persönlichem Wollen und Fühlen gesehen wird, lässt sich dies in einem Temporalgefüge darstellen. Den Untersuchungsgegenstand dieses Beitrages bildet darüber hinaus der Einfluss von den Subjunktoren während, als, wenn, seit(dem) und solange auf die zeitliche Anordnung der im Temporalsatz und im übergeordneten Satz eingeführten Situationen unter Berücksichtigung verschiedener morphologischer, syntaktischer und semantischer Faktoren.

Schlüsselwörter: temporale Relationen, Temporalsatzgefüge, partielle Gleichzeitigkeit

\section{Einleitende Bemerkungen}

Der vorliegende Beitrag traut sich an die Welt des partiellen Gleichlaufs in deutscher Sprache heran und versucht die Semantik der miteinander temporal verbundenen Ereignisse im Gleichlauf zu präsentieren, wobei der sprachliche Ausdruck der Gleichzeitigkeitsrelationen diskutiert wird. Es gibt im Deutschen zur Bezeichnung temporaler Beziehungen eine Liste von Möglichkeiten wie Partizipial-, Nominal-, Präpositional- oder Infinitivphrasen. Die temporalen Markierungen bestehen aus den Tempus-Flexionsmerkmalen am Verb, aus lexikalischen, phrasalen, sententialen und nonverbalen Möglichkeiten, mit denen Temporalität dargestellt wird: aus Adverbien (gestern, plötzlich, noch, erst, schon ...), Adjektiven (später, täglich ...), subund koordinierenden Konjunktionen (und, dann, als, nachdem, bevor, während ...), Präpositionalphrasen (vor einer Woche, am 24.12.2008 ...), den deiktischen Pronomina (ich, du, wir, ihr und der Anrede Sie), Nominalphrasen (die Bundeskanzlerin der BRD, Friedrich Schiller, die Entdeckung Amerikas 1482, der zweite Weltkrieg ...), Temporalsätzen und nonverbaler Gestik und Mimik. Jedem deutschen Verb liegt in seiner semantischen Bedeutung eine innere zeitliche Struktur zugrunde, die Aktionsart, die die Art und Weise des inneren Ablaufs eines Sachverhaltes abbildet und die interne temporale Struktur und den Charakter des jeweiligen Sachverhalts wiederspiegelt. Ein Verb wie wohnen ist eher auf Dauer angelegt, anfangen, beginnen kennzeichnen den Beginn oder beenden das Ende eines Ereignisses. Das Verb wissen ist eher statisch und Wein trinken ist ein im Moment noch nicht 
beendeter Prozess, der durch verbexterne sprachliche Elemente wie in nachdem jemand ein Glas Wein (vor 5 Minuten; leer) getrunken hat zu einem Abschluss gebracht werden kann ${ }^{2}$. Obwohl es im Deutschen die angesprochenen Möglichkeiten zur Bezeichnung temporaler Beziehungen gibt, ist das eigentliche Temporalsatzgefüge das bei weitem häufigste temporale Ausdrucksmittel.

Relative Zeitverhältnisse liegen vor, wenn zwei oder mehr verbale Sachverhalte hinsichtlich ihrer zeitlichen Einordnung und Ausführung miteinander in Beziehung gesetzt werden. Dies kann auch durch die lineare Abfolge von zwei oder mehr Handlungen innerhalb eines einzelnen Satzes bzw. im Rahmen parataktisch miteinander verbundener Sätze in Satzverbindungen angezeigt werden. Komplizierter jedoch und zugleich sprachlich relevanter ist die Beschreibung dieser Verhältnisse in Satzgefügen, vor allem in Temporalsatzgefügen, in denen unterschiedliche Arten relativer und anderer Zeitbezüge differenziert werden können. Bei den zeitlichen Verhältnissen unterscheidet man normalerweise Gleichzeitigkeit, Vorzeitigkeit und Nachzeitigkeit.

\section{Gleichzeitigkeitsrelationen}

Die Gleichzeitigkeit der Sachverhalte in beiden Teilsätzen eines Temporalsatzgefüges wird durch die den Temporalsatz einleitenden Subjunktoren während, als, wenn, seit(dem) und solange ausgedrückt, die die zeitlichen Aspekte der Gleichzeitigkeit kennzeichnen. In einem Temporalsatzgefüge der Gleichzeitigkeit überlappt sich der im übergeordneten Satz mitgeteilte Sachverhalt mit dem im Temporalsatz beschriebenen Sachverhalt. Wenn zwei Sachverhalte über annähernd dieselbe Dauer verfügen, geht man davon aus, dass es sich um ein Temporalsatzgefüge der völligen Gleichzeitigkeit handelt. Decken sich die Sachverhalte beider Teilsätze in ihrer gesamten Dauer bzw. zeitlichen Erstreckung vollkommen, so werden sie in diesen Temporalsatzgefügen als völlig gleichzeitig dargestellt. Dabei kann das zeitliche Ausmaß unterschiedlich lang sein, d.h. von einer kurzen Länge bis

\footnotetext{
2 Bei Sprachen, die eine Aspektopposition aufweisen, handelt es sich um ein morphologisches System, das die subjektive Vorstellung des Sprechers vom Verlauf, Ergebnis und Umfang der verbalen Handlung zum Ausdruck bringt. Die slawische Terminologie bezieht sich nicht auf die Verbbedeutung, sondern auf den Ablauf der Tätigkeit in der Zeit. D.h. durch den imperfektiven Aspekt wird ein Sachverhalt als geschehen, d. h. unvollendet, durch den perfektiven als geschehen und vollendet ganz ohne Rücksicht auf die Zeitstufe charakterisiert (vgl. dazu auch Wierzbicka (1999, S. 40), Wierzbicka, Schlegel (2008, S. 10f.).
} 
hin zu einer längeren Dauer. Wenn zwei Sachverhalte in einem Temporalsatzgefüge über eine unterschiedliche Dauer verfügen und darüber hinaus möglicherweise zu verschiedenen Zeiten anfangen bzw. enden, dann spricht man von partieller Gleichzeitigkeit, die in sich wiederum unterschieden werden.

Die Gleichzeitigkeit des Sachverhaltes im Temporalsatz mit dem Sachverhalt im übergeordneten Satz kann neben den Temporalsatzgefügen auch durch Partizipialkonstruktionen (Partizip Präsens) zum Ausdruck gebracht werden, die nur bei imperfektiven transitiven und intransitiven Verben auftreten. Die durch die Subjunktoren während, als und wenn eingeleiteten Temporalsätze in den Temporalsatzgefügen der Gleichzeitigkeit können in Partizipialkonstruktionen (Partizip Präsens) umgeformt werden, sofern der Temporalsatz die Handlung des Subjekts im übergeordneten Satz mitteilt wie im Beispiel (1) (vgl. Wierzbicka 2004, S.110).

(1) Ich stimmte leise summend das Tantum Ergo an, während ich mit der Brause die Schaumreste von den Wänden der sich leerenden Wanne spritzte. (BHA; 169)

=> Mit der Brause die Schaumreste von den Wänden der sich leerenden

Wanne spritzend,.....

\section{Untersuchungen zur partiellen Gleichzeitigkeit}

Bei Temporalsatzgefügen der partiellen Gleichzeitigkeit können die Temporalsätze Sachverhalte enthalten, die in ihrer Dauer dargestellt und die von den Sachverhalten in übergeordneten Sätzen unterbrochen werden. Es können verschiedene Arten von partieller Gleichzeitigkeit unterschieden werden. Partielle Gleichzeitigkeit liegt vor, wenn zwei Sachverhalte den gleichen Anfang, aber verschiedenes Ende aufweisen (vgl. (1a) und (1b)), zwei Sachverhalte verschiedenen Anfang aber gleiches Ende haben (vgl. (2a) und (2b)), ein Sachverhalt zeitlich in dem anderen enthalten ist (vgl. (3a) und (3b)), der erste Sachverhalt vor dem zweiten anfängt und vor diesem auch endet (vgl. (4a) und (4b)) oder der zweite Sachverhalt vor dem ersten Sachverhalt anfängt und endet (vgl. (5a) und (5b)).

Da der erste Sachverhalt sowohl länger (vgl. (1a - 5a)) als auch kürzer (vgl. (1b - 5b)) sein kann, so ergeben sich daraus insgesamt zehn unterschiedliche Möglichkeiten partieller Gleichzeitigkeit. 
übergeordneter Satz:

Temporalsatz:

(1b)

übergeordneter Satz:

Temporalsatz:

(2a)

übergeordneter Satz:

Temporalsatz:

(2b)

übergeordneter Satz:

Temporalsatz:

(3a)

übergeordneter Satz:

Temporalsatz:

(3b)

übergeordneter Satz:

Temporalsatz:

(4a)

übergeordneter Satz:

Temporalsatz:

(4b)

übergeordneter Satz:

Temporalsatz:

(5a)

übergeordneter Satz:

Temporalsatz:

(5b)

übergeordneter Satz:

Temporalsatz: 
Die Grenzen zwischen den während-Temporalsatzgefügen der völligen Gleichzeitigkeit und der partiellen Gleichzeitigkeit lassen sich nicht immer eindeutig ziehen. Wenn beide Sachverhalte über eine bestimmte Dauer verfügen, kann man zum einen nicht immer klar entscheiden, ob der eine Sachverhalt nur ein einziges Intervall mit dem anderen gemeinsam hat und ob beide Sachverhalte partiell gleichzeitig verlaufen, oder ob beide dieselbe Dauer aufweisen und völlig gleichzeitig bzw. simultan verlaufen. Dabei tragen auch Temporaladverbiale, die die Dauer eines der Sachverhalte begrenzen, zur richtigen Einordnung eines Temporalsatzgefüges bei. Enthält also der übergeordnete Satz eine Temporaladverbiale, dann wird wie in (1) und (2) darauf hingewiesen, dass der Sachverhalt im übergeordneten Satz nur kürzer andauert als der Sachverhalt im Temporalsatz. Die Bespiele (1) und (2) zeigen die Relation (3b).

(1) Während ich Chopin hörte, dachte ich zum ersten Mal daran, Engagements zu suchen, um ein bisschen Geld zu verdienen. (BHA; 60)

(2) Hausmeister Klapp war aufgestanden und einige Minuten neben mir gestanden, während ich telefonierte. (MHR; 13)

Wenn nun beide Sachverhalte gleichzeitig beginnen, aber der Sachverhalt im übergeordneten Satz durch ein Temporaladverbiale begrenzt wird und vor dem Sachverhalt im Temporalsatz endet, dann liegt im Beispiel (3) ebenfalls die Relation (1b) vor.

(3) Auch Siddhartha fühlte Sehnsucht und den Quell des Geschlechts sich bewegen; da er aber noch nie ein Weib berührt hatte, zögerte er einen Augenblick, während seine Hände schon bereit waren, nach ihr zu greifen. (HHS; 45)

Durch ein während-Temporalsatzgefüge werden sprachlich nur solche Relationen zum Ausdruck gebracht, in denen der Sachverhalt im übergeordneten Satz völlig gleichzeitig mit dem Sachverhalt im Temporalsatz verläuft, oder aber in diesem enthalten ist, d.h. also die Relationen (2b) und $(3 b)^{3}$. Darüber hinaus sind auch die Relationen (4a), (5a), (4b) sowie (5b)

\footnotetext{
3 Vgl. dazu auch zu den anderen Ergebnissen für während Ballweg (1988, S.166ff.), Eisenberg (1986, S. 352f.), Herweg (1990, S. 260f.) und Lutzeier (1981), die bei den durativen Sachverhalten in beiden Teilsätzen nur die Überlappung von den Sachverhalten für notwendig halten. Dabei wird auch gefordert, dass in einem Temporalsatzgefüge der längere Sachverhalt den kürzeren enthält, unabhängig davon, ob im übergeordneten Satz bzw. im Temporalsatz.
} 
zugelassen, sofern Sachverhalte in beiden Teilsätzen nicht über dieselbe Dauer verfügen, was ebenfalls möglich ist (vgl. (4)).

(4) Die Häuser ihrer Arbeitgeber sind schon gecheckt worden, wir haben in ihrem Heimatdorf Recherchen angestellt, die Wohnung von Frau Woltersheim ist, während sie hier vernommen wurde, noch einmal gründlich untersucht worden. (BHV; 66)

Da die Erzählung wohl länger als die Tätigkeit im Temporalsatz dauert, ist Beispiel (5) ein Beleg für die Relation (2a) bzw. (3a). Darüber hinaus kann die Relation (3a), bei der der Sachverhalt im übergeordneten Satz den Sachverhalt im Temporalsatz mit einschließt, auch in folgenden Belegen vorliegen (vgl. (6)).

(5) Sie holt eine Gulaschsuppe aus der Küche am anderen Ende des Tresens und setzt von dort aus, während sie Brot und Besteck auf den Unterteller legt, ihre Erzählung fort. (SPM; 63)

(6) Ihr Blick blieb verständnislos, während ihre fahl werdenden Lippen sich unnütz und hilflos öffneten und schlossen. (SVD; 8)

Dabei scheint nicht nur die Tatsache relevant $\mathrm{zu}$ sein, dass der Sachverhalt im übergeordneten Satz auch schon vor oder noch nach dem Verhalt im Temporalsatz stattgefunden hat, sondern auch, dass die Dauer des Sachverhaltes im Temporalsatz in den Blick genommen wird und darüber hinaus lediglich die Aussage über die Zeit gemacht wird, die durch den Sachverhalt im Temporalsatz bezeichnet wird. Dabei wird sprachlich nur mitgeteilt, dass der Sachverhalt im übergeordneten Satz innerhalb dieser Zeit verläuft. Dies gilt auch für die Fälle, in denen durch eine zusätzliche Angabe bzw. durch die Verbsemantik explizit ausgesagt wird, dass der Sachverhalt im übergeordneten Satz auch vor bzw. noch nach, oder aber vor und nach dem Verhalt im Temporalsatz stattfindet (vgl. (7) und (8)).

(7) Während ich dies schreibe, liegt sie vor mir auf dem Tisch und sieht aus großen gelben Augen über meine Schulter auf einen Fleck der Wand. Dreimal hab ich mich schon danach umgedreht und kann dort nichts sehen als das alte dunkle Holz. (HMW; 41)

(8) Als er dann aufstand [...]die Brandung des Meeres hörte, da kannte seine gute Laune keine Grenzen mehr, und er spitzte die Lippen und pfiff eine muntere Melodie. Während er sich ankleidete, pfiff er weiter und pfiff immer noch, als er sein Zimmer verließ und mit beschwingtem Schritt über den Gang an die Kammertüre seiner Tochter trat. (SPP; 281) 
In den seit-Temporalsatzgefügen der partiellen Gleichzeitigkeit, in denen die Sachverhalte beider Teilsätze nur ein Teilintervall gemeinsam haben, wird einerseits zum Ausdruck gebracht, dass der Sachverhalt im übergeordneten Satz irgendwann innerhalb der durch den Sachverhalt im Temporalsatz bereits eingenommenen Zeitspanne begonnen hat und zur Betrachtzeit noch andauert. Dabei gilt der Sachverhalt im Temporalsatz, der wie der Sachverhalt im übergeordneten Satz über eine gewisse Dauer verfügt und eine Zeitlang parallel zum Sachverhalt im übergeordneten Satz verlief, zur Betrachtzeit nicht mehr. In den meisten Fällen liegt kein gleichzeitiger Beginn der Teilsatzsachverhalte vor, sondern der Sachverhalt im übergeordneten Satz fängt erst an, nachdem der Sachverhalt im Temporalsatz bereits eine Zeitlang besteht (9).

(9) Der Gedanke an eine sommerliche Übersiedlung auf die Alm beschäftigte mich ganze Abende lang. Weil ich, seit ich weniger im Freien arbeitete, schlechter schlief, blieb ich abends länger auf [...] und las in Luises Magazinen, den Kalendern und Kriminalromanen. (HMW; 106)

Die richtige Interpretation der seit-Temporalsätze ist durch den Kontext gesichert. Es kann aber auch von einer temporalen Ambiguität ausgegangen werden. In (10) handelt es sich um eine partielle Gleichzeitigkeit. Dabei kann bei geeignetem Kontext entweder das Zeitverhältnis der völligen Gleichzeitigkeit (seit Beginn unseres Aufenthalts) oder das der partiellen Gleichzeitigkeit (nach unserem Aufenthalt) zugelassen werden.

(10) Poznań war eine ungeheuer saubere und ordentliche Stadt. Die Leute in der Straßenbahn waren vorwiegend ernst. Ich hatte das Gefühl, seit wir in Poznań waren, wurde Jan vollkommen anders. (SRR; 141)

Dabei kann bei einem geeigneten Kontext einerseits entweder das Zeitverhältnis der völligen Gleichzeitigkeit oder aber das der partiellen Gleichzeitigkeit zugelassen werden. Von partieller Gleichzeitigkeit kann dann gesprochen werden, wenn der Sachverhalt im übergeordneten Satz ansetzt, nachdem der Sachverhalt im übergeordneten Satz nach dem Abschluss des Sachverhaltes im Temporalsatz schon eine gewisse Weile stattfindet oder aber zur Betrachtzeit andauert, während der Sachverhalt im Temporalsatz abgeschlossen ist, d.h. Jan befindet sich zur Betrachtzeit nicht mehr in Poznań, hat sich aber auch schon vollkommen verändert, als er noch in Poznań war. Von der völligen Gleichzeitigkeit wird wiederum dann die Rede sein, wenn der Sachverhalt im Temporalsatz und der Sachverhalt im übergeordneten Satz gemeinsam ansetzen und bis zur Betrachtzeit andauern, d.h. Jan befindet sich 
zur Betrachtzeit in Poznań und macht, seit er dort ist, einen veränderten Eindruck. Andererseits kann durch einen bestimmten Kontext das Zeitverhältnis der Vorzeitigkeit vorliegen, wenn der Sachverhalt im übergeordneten Satz nach dem Abschluss des Sachverhaltes im Temporalsatz beginnt und zur Betrachtzeit andauert, d.h. Jan ist zur Betrachtzeit nicht mehr in Poznań und macht erst nach seiner Rückkehr aus Poznań einen vollkommen veränderten Eindruck.

In den solange-Temporalsatzgefügen verfügen sowohl beide Teilsatzsachverhalte über eine gewisse Dauer, die jedoch nicht unbegrenzt andauern kann. Alle Sachverhalte mit unbegrenzter Dauer bzw. irreversible Zustände wie beispielsweise tot sein, die nicht unterteilbar sind und über keinen Endpunkt verfügen, sind von vornherein in den solangeTemporalsatzgefügen ausgeschlossen (11).

(11) Solange ich atme, hoffe ich. (dum spiro spero, lat., philos. Weisheit)

(11') *Solange ich nicht atme...

In den solange-Temporalsatzgefügen sind kurzzeitige Sachverhalte dann zugelassen, wenn sie negiert oder wiederholbar sind. Bei wiederholbaren Sachverhalten in beiden oder auch nur in einem der Teilsätze bildet die Summe der Wiederholungen eine mit dem Sachverhalt des jeweils anderen Teilsatzes parallel verlaufende Zeitspanne (12).

(12) Oma Hela ist ihr Leben lang jeden Sonntag in die erste Messe gegangen, [...], sie war ungeheuer katholisch und fromm, aber sie hat nie, solange sie in Berlin gewohnt hat, und das waren bald dreißig Jahre, niemals in dieser Zeit, sage ich, hat sie einen Pfennig Kirchensteuer bezahlt. (SRR; 17).

Durch die Negation im übergeordneten und/oder im Temporalsatz wird ausgedrückt, dass die Dauer der Nicht-Ausführung des einen Sachverhalts parallel ist mit der Dauer der Ausführung des anderen bzw. bei Negation der beiden Teilsätze als Dauer der Nicht-Ausführung des anderen Sachverhalts aufzufassen ist. Dies gilt auch dann, wenn die Sachverhalte beider Teilsätze auch ohne Negation durativ sind. Negierte kurzzeitige Sachverhalte können sich wie durative Sachverhalte verhalten, was bei den solangeTemporalsatzgefügen sowohl für den Sachverhalt im übergeordneten (13) als auch für den im Temporalsatz (14) gilt. 
(13) Er wollte sie nicht mehr aus seinem Zimmer lassen, wenigstens nicht, solange er lebte, seine Schreckgestalt sollte ihn zum ersten Mal nützlich werden. (KFV; 99)

(14) Und solange er nicht im Clinch mit irgendeiner (für die Presse) heißen

Tussi fotografiert wurde, konnte ihnen nichts passieren. (RIS; 49).

Es kann auch vorkommen, dass die Sachverhalte im übergeordneten und im Temporalsatz nicht völlig gleichzeitig verlaufen und der Sachverhalt im übergeordneten Satz, der mitunter kurz sein kann, in einem zeitlichen Rahmen ausgeführt wird, der durch den Sachverhalt im Temporalsatz gesetzt wird. Demzufolge wird durch den Temporalsatz in (15) nicht angegeben, wie lange sie wirklich duscht. D.h. es wird nicht mitgeteilt, ob sie bis zum Abstellen des Wasserhahns duscht oder bis kaltes Wasser nachfließt.

(15) Solange sich diese Irritation auf die Deutschen hinter der Mauer beschränkte, war sie kaum mehr als ein Besuchserlebnis. (SPM; 13).

Darüber hinaus können in einem Temporalsatz Adverbien wie noch erscheinen, durch die ausgedrückt wird, dass der Sachverhalt im Temporalsatz zwar zur Betrachtzeit noch gilt, aber sein Ende möglicherweise bevorsteht. In (16) wird durch den Temporalsatz angegeben, wie viel Zeit zur Ausführung des Sachverhalts im übergeordneten Satz nach Ansicht des Sprechers noch verbleibt.

(16) Meine gesamte einschlägige Erfahrung bestand darin, daß ich, solange ich noch zur Penne ging, aufgewärmt hatte, was mir nach den Einfällen meiner Greisin als Mittagessen zugedacht war. (SRR; 218)

In den wenn/als-Temporalsatzgefügen der partiellen Gleichzeitigkeit wird einerseits zum Ausdruck gebracht, dass der Sachverhalt im übergeordneten Satz irgendwann innerhalb der durch den Sachverhalt im Temporalsatz bereits eingenommenen Zeitspanne begonnen hat und zur Betrachtzeit noch andauert (17) und (18).

(17) Wenn man ihm in die Augen sieht, könnt' man das andere an ihm glatt vergessen. (GGB; 537)

(18) Als ich neunzehn war, stand meine innere Welt über Nacht fest. Durch den Erfolg von Probefotos. (KBI; 375) 
Durch die Altersangaben wie beispielsweise Jahre alt bzw. jung/klein/Kind sein die im Temporalsatz erscheinen, kann zumeist nicht aktuell-prozedente Bedeutung, sondern in der Regel eine längere Zeitperiode bezeichnet werden, innerhalb derer der Sachverhalt im übergeordneten Satz verläuft. Der in seiner Dauer und in seinem Nicht-Abschluss mitgeteilte Sachverhalt kann in Form einer Altersangabe eine Lebens- bzw. Tätigkeitsperiode bezeichnen, innerhalb derer der zweite, abgeschlossene Sachverhalt stattfindet (18).

(18) Er starb, als ich sechs Jahre alt war, im Alter von siebenunddreißig Jahren an einer Lungenverletzung, die er im Krieg erlitten hatte. (BHV; 20)

Um das Fortschreiten des übergeordneten Satzes bzw. des Temporalsatzes zu kennzeichnen, erscheinen im als/wenn-Temporalsatzgefüge mitunter gerade, dabei sein, etwas zu tun oder am Tun sein (19) und (20). Dadurch wird zudem der Realisierungsverlauf des Sachverhalts hervorgehoben.

(19) In Rosas Dunkelkammer entwickelte ich die Filme und als ich gerade dabei war, die Ausschnitte zu wählen und sie zu vergrößern, klingelte das Telefon. (SVD; 23)

(20) Ich wollte gerade weiterblättern, als jemand an die Tür klopfte. (MHR; $10)$.

\section{Abschließende Bemerkungen}

Das Temporalsatzgefüge hat nahezu unbeschränkten Anwendungsbereich, der von dem fast nur angedeuteten Zusammenhang bis zur zwingenden Notwendigkeit, von Allgemeinaussagen und didaktischen Erläuterungen bis zu emotional bestimmten Motivationen reicht. Wo immer ein temporaler Zusammenhang zwischen Tatsachen, Ereignissen, Handlungen und Verhältnissen oder auch zwischen persönlichem Wollen und Fühlen gesehen wird, lässt sich dies in einem Temporalgefüge darstellen. Allgemein gilt, dass in komplexen Sätzen, die aus je mindestens zwei satzartigen Konstruktionen bestehen, zwei Sachverhalte zueinander in Beziehung gesetzt werden.

In dem vorliegenden Beitrag wurden anhand von standardsprachlichen deutschen Quellentexten aus der Belletristik einerseits der Zeitstufenbezug und das gegenseitige Verhältnis der Tempora, die als Zeitinformatoren gelten, in den Temporalsatzgefügen der partiellen Gleichzeitigkeit im Deutschen untersucht und der Zeitwert der relativen Tempora, also unter anderem ihre 
zeitliche Leistung, andererseits überprüft und diskutiert. Es wurde drüber hinaus gezeigt, dass sich Grenzen zwischen den Temporalsatzgefügen der völligen Gleichzeitigkeit und der partiellen Gleichzeitigkeit sich nicht immer eindeutig und nur kontextbedingt ziehen lassen. Wenn beide Sachverhalte über eine gewisse Duration verfügen, kann man zum einen nicht immer klar entscheiden, ob der eine Sachverhalt nur ein Intervall mit dem anderen gemeinsam hat und ob beide Sachverhalte partiell gleichzeitig verlaufen, oder aber zum anderen, ob beide dieselbe Duration aufweisen und völlig gleichzeitig, also simultan verlaufen.

\section{QUELLENVERZEICHNIS}

BHA Böll, Heinrich (1992). Ansichten eines Clowns. Köln: Kiepenheuer \& Witsch Verlag.

BHV Böll, Heinrich (1991). Die verlorene Ehre von Katharina Blum, oder Wie Gewalt entstehen und wohin sie führen kann. Köln: Kiepenheuer \& Witsch Verlag.

GGB Grass, Günter (1992). Die Blechtrommel. Roman. In: Günter Grass: Werkausgabe in zehn Bänden, Bd. II. Darmstadt und Neuwied: Luchterhand Verlag.

HHS Hesse, Hermann (1982). Siddhartha. Eine indische Erzählung. Frankfurt am Main: Suhrkamp Taschenbuch Verlag.

HMW Haushofer, Marlen (1995). Die Wand. Stuttgart: Klett Verlag.

KBI Kirchhoff, Bodo (1991). Infanta. Frankfurt am Main: Suhrkamp Verlag.

KFV Kafka, Franz. (1983). Die Verwandlung. Frankfurt am Main: Fischer Taschenbuch Verlag.

MHR Martin, Hansjörg (1988). Der Rest ist Sterben. Reinbek bei Hamburg: Rowohlt Verlag.

RIS Rodrian, Irene. (1992). Strandgrab. Reinbek bei Hamburg: Rowohlt Verlag.

SPM Schneider, Peter (1991). Der Mauerspringer. Hamburg, Zürich: Luchterhand Literaturverlag.

SRR Schneider, Rolf (1975). Die Reise nach Jaroslav. Darmstadt und Neuwied: Luchterhand Verlag.

SPP Süskind, Patrick (1990). Das Parfüm. Die Geschichte eines Mörders. Frankfurt am Main: Gutenberg Verlag.

SVD Schatten, Viola (1991). Dienstag war die Nacht zu kurz. Frankfurt am Main: Fischer Verlag.

\section{LITERATURVERZEICHNIS}

Ballweg, J. (1988). Die Semantik der deutschen Tempusformen. Eine indirekte Analyse im Rahmen einer temporal erweiterten Aussagenlogik. Sprache der Gegenwart 70. Düsseldorf: Schwann Verlag. 
Eisenberg, P. (1999). Grundriß der deutschen Grammatik. Bd.2: Der Satz. Stuttgart/Weimar: J.B. Metzlersche Verlagsbuchhandlung.

Herweg, M. (1990). Zeitaspekte. Bedeutung von Tempus, Aspekt und temporalen Konjunktionen. Wiesbaden: Deutscher Universitätsverlag.

Lutzeier, P. R. (1981). Wahrheitsdefinitorische Überlegungen zur temporalen Lesart der Konjunktion „während“. In: Linguistische Berichte 76, 1-24. Wiesbaden: Viehweg \& Sohn Verlag.

Wierzbicka, M. (1999). Das Tempus- und Aspektsystem im Deutschen und Polnischen. Versuch einer integralen Theorie im Rahmen einer temporal erweiterten Aussagenlogik. Sammlung Groos Bd. 70. Heidelberg: Groos Verlag.

Wierzbicka, M. (2004). Zeitbeziehungen in den Temporalsatzgefügen erörtert an den Gegebenheiten der Consecutio Temporum im Deutschen und im Polnischen. München: Iudicium Verlag.

Wierzbicka, M., Schlegel, D. (2008). Sprechzeiten im Diskurs. Zum absoluten und relativen Gebrauch der Tempora in der gesprochenen deutschen Sprache. München: Iudicium Verlag. 
